\title{
Criteria for Inclusion of Newer Bariatric and Metabolic Procedures into the Mainstream: a Survey of 396 Bariatric Surgeons
}

\author{
Kamal K. Mahawar ${ }^{1}$ - Cynthia-Michelle Borg ${ }^{2}$ - Sanjay Agarwal ${ }^{3}$ - Rui Riebeiro ${ }^{4}$. \\ Maurizio De Luca ${ }^{5}$ Peter K. Small ${ }^{1}$
}

Published online: 5 October 2016

(C) Springer Science+Business Media New York 2016

\begin{abstract}
Background There is currently no consensus on the criteria for inclusion of new bariatric procedures into routine clinical practice. This study canvasses bariatric surgeons in an attempt to define these criteria.

Methods Bariatric Surgeons from around the world were invited to participate in a questionnaire-based survey on SurveyMonkey ${ }^{\circledR}$.

Results 396 bariatric surgeons, 337 International Federation for the Surgery of Obesity and Metabolic Disorders (IFSO) members, took the survey. Five clinical studies conducted under the strict monitoring of an Institutional Review Board would satisfy most surgeons $(67.7 \%, n=266)$. When asked regarding the number of patients in these studies, a cumulative number of 500 patients would satisfy $64.5 \%(n=255)$ of the surgeons. Most respondents regarded endorsement by their national society and IFSO as 'very important' or 'extremely important'. An overwhelming $74.4 \%(n=294)$ felt that every new procedure should undergo a randomized comparison against one of the established alternatives like Roux-en-Y Gastric Bypass or Sleeve Gastrectomy.
\end{abstract}

Kamal K. Mahawar

kamal_mahawar@hotmail.com

Bariatric Unit, Sunderland Royal Hospital, Sunderland SR4 7TP, UK

2 Lewisham and Greenwich NHS Trust, University Hospital Lewisham, London, UK

3 Homerton University Hospital, London, UK

4 Centro Hospitalar de Lisboa Central, Unidade de Tratamento Cirúrgico da Obesidade e Doenças Endoórinas, Lisbon, Portugal

5 Obesity Unit, Montebelluna Treviso Hospital, Veneto, Italy
Conclusion Evaluation of a new bariatric procedure in at least 5 adequately supervised clinical studies (four of which must be randomized comparisons with one of the existing alternatives) reporting at least 5 years results on a minimum of 500 patients would satisfy majority of bariatric surgeons for the inclusion of a new bariatric procedure into clinical practice. The findings of this survey are simply aimed at starting a discussion on this topic and cannot be used to influence the ground reality until an international consensus can be reached amongst experts.

Keywords Experimental procedures $\cdot$ New procedures . Bariatric surgery $\cdot$ Obesity surgery $\cdot$ One anastomosis gastric bypass $\cdot$ Mini gastric bypass

\section{Introduction}

Driven by a desire to develop safer, better, and more durable alternatives to the existing options, bariatric Surgery is witnessing innovation at a rapid rate. There is however currently no consensus on the criteria for inclusion of a new bariatric procedure into routine clinical practice. On one hand, this risks adoption of new experimental procedures before demonstrated clinical outcomes; on the other, this may lead to undue delays even in the face of robust data. In the absence of clear guidance, endorsement decisions by national and international bariatric societies could also be arbitrary depending on the views of the position holders.

Surgical innovation generally poses a number of ethical dilemmas [1]. But when it comes to bariatric surgery, the challenge is even more intimidating, as there are potentially a large number of outcome measures each procedure could be assessed upon [2]. Adoption of new procedures into clinical practice before demonstrated outcomes may put patients at 
unnecessary risk as well as bring the specialty into disrepute. This has led many authoritative voices to express caution [3, 4] against unregulated over enthusiastic innovation.

Bariatric surgeons are however not new to moral and ethical challenges $[5,6]$. Over the last couple of decades, the specialty has fought off a few such dilemmas to come to be recognized as a serious scientific discipline. Though it would appear contradictory, development of clear processes that a new bariatric surgical procedure should go through prior to its acceptance into the mainstream bariatric practice will eventually facilitate innovation and protect both patients and surgeons.

The purpose of this study was to survey the opinion of the global community of bariatric and metabolic surgeons on the process of development of new bariatric and metabolic procedures and criteria that should be met before a new bariatric or metabolic procedure could be introduced into routine clinical practice.

\section{Methods}

A questionnaire-based survey (https://www.surveymonkey. co.uk/r/ZCSMV87) was conducted of the global community of bariatric and metabolic surgeons. Surgeons were invited to take the survey on SurveyMonkey ${ }^{\circledR}$ starting on 12th March' 2016. The link to the survey was freely shared on the social media, email chat group of surgeons, and through personal networks. An email was also sent to presidents of all the national bariatric societies affiliated to International Federation for the Surgery of Obesity and Metabolic Disorders (IFSO) for circulation amongst their membership. The survey was closed for analysis on 20th April 2016. Basic descriptive statistics was used. This study was only designed to survey the opinion of bariatric surgeons on this topic. It is not an attempt at building consensus.

The choices offered in the survey were felt to be the most logical options by the authors. To ensure that respondents could also enter other options, we also provided a free text option in each and every question.

\section{Results}

A total of 422 individuals participated in the survey. Out of these 396 described themselves as a bariatric/metabolic surgeon. Further analysis was only carried out on these 396 individuals only and the data for others was discarded. Table 1 shows the country of origin of the respondents with the percentage and the actual numbers.

Of these $337(85.5 \%)$ surgeons were members of IFSO and $95.7 \%(378 / 395)$ were members of a national society affiliated to IFSO. A total of 139 (36.1 \%)
Table 1 Country of Origin of Respondents in Alphabetical Order

\begin{tabular}{|c|c|c|}
\hline Country of Origin & Percentage & $\begin{array}{l}\text { Number of } \\
\text { Responses }\end{array}$ \\
\hline Argentina & $2.3 \%$ & 9 \\
\hline Australia & $3.8 \%$ & 15 \\
\hline Austria & $0.5 \%$ & 2 \\
\hline Belgium & $2.8 \%$ & 11 \\
\hline Brazil & $9.2 \%$ & 36 \\
\hline Canada & $0.3 \%$ & 1 \\
\hline Chile & $3.1 \%$ & 12 \\
\hline China & $0.3 \%$ & 1 \\
\hline Colombia & $2.8 \%$ & 11 \\
\hline Czech Republic & $1.0 \%$ & 4 \\
\hline Dominican Republic & $0.8 \%$ & 3 \\
\hline Egypt & $2.6 \%$ & 10 \\
\hline France & $3.6 \%$ & 14 \\
\hline Germany & $1.3 \%$ & 5 \\
\hline Greece & $1.5 \%$ & 6 \\
\hline India & $12.0 \%$ & 47 \\
\hline Israel & $0.5 \%$ & 2 \\
\hline Italy & $6.9 \%$ & 27 \\
\hline Jordan & $0.3 \%$ & 1 \\
\hline Kuwait & $0.8 \%$ & 3 \\
\hline Lebanon & $1.5 \%$ & 6 \\
\hline Mexico & $2.8 \%$ & 11 \\
\hline Netherlands & $1.0 \%$ & 4 \\
\hline New Zealand & $0.5 \%$ & 2 \\
\hline Nicaragua & $0.3 \%$ & 1 \\
\hline Norway & $0.5 \%$ & 2 \\
\hline Pakistan & $0.3 \%$ & 1 \\
\hline Panama & $0.3 \%$ & 1 \\
\hline Paraguay & $0.5 \%$ & 2 \\
\hline Peru & $0.5 \%$ & 2 \\
\hline Philippines & $0.3 \%$ & 1 \\
\hline Poland & $0.8 \%$ & 3 \\
\hline Portugal & $3.6 \%$ & 14 \\
\hline Republic of Korea & $0.3 \%$ & 1 \\
\hline Romania & $0.3 \%$ & 1 \\
\hline Russian Federation & $0.8 \%$ & 3 \\
\hline Saudi Arabia & $1.3 \%$ & 5 \\
\hline Singapore & $0.5 \%$ & 2 \\
\hline South Africa & $0.3 \%$ & 1 \\
\hline Spain & $5.6 \%$ & 22 \\
\hline Sweden & $1.5 \%$ & 6 \\
\hline Switzerland & $1.0 \%$ & 4 \\
\hline Turkey & $0.3 \%$ & 1 \\
\hline Ukraine & $0.8 \%$ & 3 \\
\hline United Arab Emirates & $1.8 \%$ & 7 \\
\hline United Kingdom & $11.0 \%$ & 43 \\
\hline United States of America & $5.4 \%$ & 21 \\
\hline Venezuela (Bolivarian Republic of) & $0.3 \%$ & 1 \\
\hline
\end{tabular}


respondents regarded themselves as a 'key opinion maker' and a further $81(21 \%)$ respondents were not sure if they were a key opinion maker whereas 165 (42.8\%) respondents did not belong to this category. A 'key opinion maker' was loosely defined as somebody with a position on the national/international organizations or committees, professorships, serious researcher etc. Amongst the survey respondents were 4 IFSO presidents, 3 presidents of American Society for Metabolic and Bariatric Surgery (ASMBS), and 4 presidents of national societies.

\section{Number of Cohort Studies, Patients, and Follow up}

Table 2 gives an idea of the number of 'studies' that the respondents felt should be conducted on human subjects under careful monitoring of an Institutional Review Board (IRB) before a new procedure can be regarded as an accepted treatment option. A significant $67.68 \%(n=266 / 393)$ of the respondents felt $\leq 5$ studies were sufficient for this purpose, and another $12.97 \%$ ( $n=51 / 393)$ surgeons felt that a minimum of 10 studies should be required. Interestingly $9.41 \%(n=37 /$ 393) surgeons wanted $\geq 20$ studies.

Table 3 presents data on the number of 'patients' that the respondents felt any new procedure must first be performed on, under the careful monitoring of an IRB before it can be regarded as an accepted treatment option. Out of 395 respondents who answered this question, $64.5 \%(n=255)$ felt that any new procedure must first be performed on $\leq 500$ procedures before it can be regarded as an accepted treatment. Only $11.9 \%$ of the respondents $(n=47)$ felt that every new procedure should first be performed on $>1000$ patients.

Table 4 details the mandatory follow-up period under careful monitoring. Most of the respondents would be satisfied with 5 years follow-up $(n=336,85.5 \%)$. Interestingly 28 (7.1\%) respondents wanted the follow-up duration to be 10 years or longer.

Table 2 Number of Studies under careful monitoring of an Institutional Review Board needed

\begin{tabular}{lcr}
\hline $\begin{array}{l}\text { Number of Studies } \\
\text { Under Monitoring } \\
\text { of an IRB }\end{array}$ & $\begin{array}{l}\text { Number of Responses } \\
(\mathrm{n}=393)\end{array}$ & Percentage \\
\hline 1 & 7 & $1.78 \%$ \\
2 & 28 & $7.12 \%$ \\
3 & 76 & $19.34 \%$ \\
4 & 19 & $4.83 \%$ \\
5 & 136 & $34.61 \%$ \\
$>5$ & 127 & $32.32 \%$ \\
\hline
\end{tabular}

Table 3 Number of Patients under careful monitoring of an Institutional Review Board needed

\begin{tabular}{llr}
\hline $\begin{array}{l}\text { Number of Patients Under } \\
\text { Monitoring of an IRB }\end{array}$ & $\begin{array}{l}\text { Number of Responses } \\
(\mathrm{n}=395)\end{array}$ & Percentage \\
\hline $1-50$ & 21 & $5.3 \%$ \\
$51-100$ & 78 & $19.7 \%$ \\
$101-250$ & 77 & $19.5 \%$ \\
$251-500$ & 79 & $20.0 \%$ \\
$501-1000$ & 93 & $23.5 \%$ \\
$>1000$ & 47 & $11.9 \%$ \\
\hline
\end{tabular}

\section{Endorsement by National Society and IFSO}

Figure 1 gives us an idea of how important is the endorsement by the respondent's national society to them where 'Extremely Important' meant that the respondent would not perform a new procedure without the endorsement of his/her national society. Out of 393 respondents who answered this question, $36.1 \%(n=142)$ and $41.7 \%(n=164)$ respondents regarded endorsement by their national society as 'extremely important' and 'very important' respectively.

Figure 2 presents data on the importance of IFSO endorsement for respondents. Out of 394 respondents who answered this question, $29.4 \%(n=116)$ and $44.4 \%(n=175)$ respondents regarded IFSO endorsement as 'extremely important' and 'very important' respectively.

\section{Randomized Controlled Trials}

An overwhelming $74.4 \%(n=294)$ felt that every new procedure should first be examined against one of the standard bariatric procedures in a randomized setting. Only $15.7 \%$ $(n=62)$ did not feel it to be a necessary prerequisite and $9.9 \%(n=39)$ were 'not sure'.

Those respondents who wanted such randomized comparisons were then asked further questions regarding which

Table 4 Follow-Up Duration under careful monitoring of an Institutional Review Board needed

\begin{tabular}{lcc}
\hline $\begin{array}{l}\text { Mandatory Follow Up Under } \\
\text { Monitoring of an IRB }\end{array}$ & $\begin{array}{l}\text { Number of Responses } \\
(\mathrm{n}=393)\end{array}$ & Percentage \\
\hline$<6$ months & 0 & $0.0 \%$ \\
$6-12$ months & 11 & $2.8 \%$ \\
12-18 months & 26 & $6.6 \%$ \\
18-24 months & 65 & $16.5 \%$ \\
2-3 years & 77 & $19.6 \%$ \\
$3-4$ years & 24 & $6.1 \%$ \\
$4-5$ years & 133 & $33.8 \%$ \\
$>5$ years & 57 & $14.5 \%$ \\
\hline
\end{tabular}


Fig. 1 How Important is Endorsement by National Society?

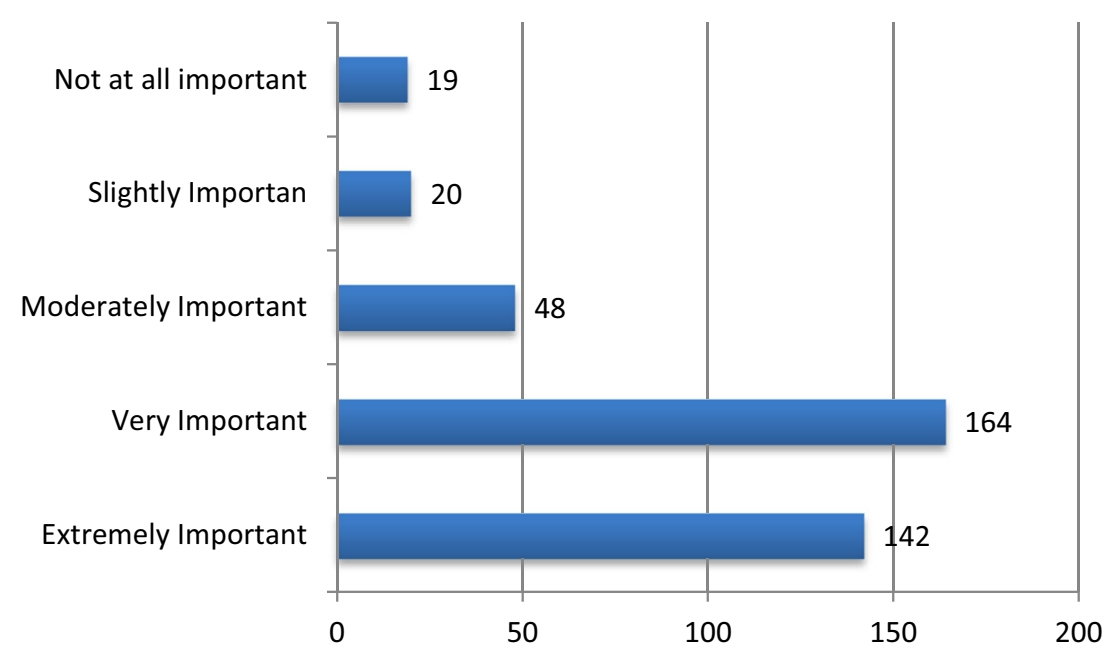

procedure should a new procedure be compared against it and how many such comparisons were needed. A total of 317 respondents answered the question on the most appropriate existing procedure for randomized comparison with the new one. Respondents were allowed to select more than one procedure. Out of these, 267 respondents $(84.23 \%)$ felt every new procedure should be examined against Roux-en-Y Gastric Bypass (RYGB); 174 (54.9\%) said Sleeve Gastrectomy (SG), 64 (20.2\%) said One Anastomosis (Mini) Gastric Bypass and 25 (7.9\%) said Gastric Banding. Thirty $(9.4 \%)$ respondents wanted a new procedure compared against 'other procedures' like Biliopancreatic Diversion/ Duodenal Switch (7/317), Gastric Plication (5/317), Ileal Transposition etc.

When asked regarding the number of Randomized Controlled Trials, 309 respondents answered the question. The majority of these respondents $(54.4 \%, n=168)$ would be satisfied with 4 or less randomized studies. Another large group $(30.7 \%, n=95)$ would like to have 5 such randomized comparisons. A smaller percentage $(14.9 \%, n=46)$ wanted

Fig. 2 How Important is Endorsement by IFSO? more than 5 randomized studies. Half of these respondents ( $n=23,7.5 \%)$ wanted 10 trials.

A total of 317 respondents answered the question regarding which outcome measures should be studied in such randomized comparisons. Respondents were allowed to select more than one response. Table 5 presents the outcome measures with the number of respondents voting for it.

\section{Comments}

We also asked respondents to provide "any other comment" at the end of the questionnaire. An attempt was then made to identify and develop important themes from these comments using a deductive approach.

1. One of the themes that emerged was that the quality of the studies was as important as the quantity embodied in the comments like:

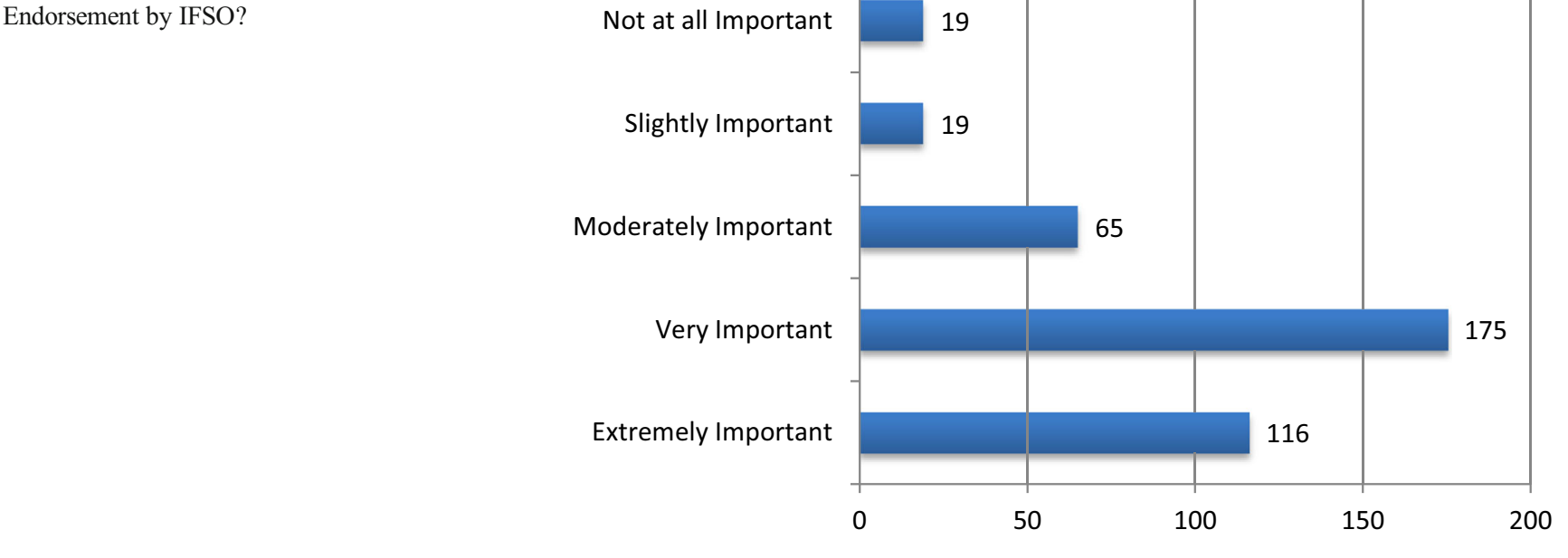


Table 5 Outcome Measures for Study in Randomized Comparisons

\begin{tabular}{lll}
\hline Outcome Measure & $\begin{array}{l}\text { Number of } \\
\text { Responses } \\
(n=393)\end{array}$ & Percentage \\
\hline Weight Loss & 283 & $89.3 \%$ \\
30-day morbidity and mortality & 252 & $79.5 \%$ \\
Longer term morbidity and mortality & 258 & $81.4 \%$ \\
Impact on co-morbidities like Type 2 & 273 & $86.1 \%$ \\
$\quad$ Diabetes Mellitus & 265 & $83.6 \%$ \\
Quality of Life of Patient & 180 & $56.8 \%$ \\
Cost Effectiveness & 44 & $13.9 \%$ \\
Any other outcome measure(s) & &
\end{tabular}

"Even one adequately powered, multi-centre randomized controlled trial with adequate follow-up should be enough".

"The studies have to come from proper academic centres" again emphasizing that the quality of the study was as important as the quantity.

2. Some respondents highlighted the necessity for prior animal studies before human trials.

"Preclinical animal model studies are needed before human studies".

3. A number of surgeons were concerned regarding lack of monitoring of the studies.

"Too many people are experimenting in Bariatric Surgical procedures." Table 6 presents some other useful comments (edited) relevant to the survey.

\section{The Questionnaire}

Finally, Table 7 provides a brief questionnaire with numbers of individual responses for each question.

\section{Discussion}

Despite authoritative voices expressing concerns against unethical behaviour and unregulated innovation in the field of bariatric surgery $[3,4]$, there is as yet no attempt in scientific literature to define the process for inclusion of a new procedure into the mainstream bariatric practice. This survey is the first attempt to start a discussion on the proposed criteria for such benchmarking.

For a relatively small specialty, this survey of 396 bariatric surgeons from around the world represents a large sample. As can be seen in Table 1, respondents in this study represent almost every single country where bariatric surgery is performed. Though numbers of respondents are not always in proportion to the number of procedures performed in a country, authors cannot think of any group of surgeons with a strong interest in the area that would be more or less likely to take part. It provides several useful indicators for national societies and IFSO to use for indicative purposes while benchmarking individual bariatric procedures for their membership. As expected, the majority of surgeons viewed endorsements by their national societies and IFSO as 'very important' or 'extremely important'. This must place additional responsibility on the office bearers in these institutions to ensure that any evaluation follows a scientific process.

Table 6 Some Other Interesting Comments (Edited)

The quality of the studies is more important than quantity.

Studies should be carried out in different geographical areas separately.

Even many existing procedures have not been evaluated fully yet.

The IRB is a local thing and varies from country to country. What some consider experimental others do not.

Societies should have a technical committee to assess the technical and historical aspects of new procedures, and if not very different from existing ones, it should be cleared for faster acceptance.

I think we need to differentiate procedures that are very similar and use the same concepts of gastric reduction and small bowel shortening from those that use other mechanisms.

I do not believe that organizations such as the ASMBS or IFSO should 'endorse' procedures. I do not feel that organizations should be regulatory, but advisory to its members and society.

It is important to consider if a new procedure can have dangerous implications.

RCT is important to standardize the new procedure, but not perform it.

I would like to see the endoscopic bariatric procedures included in the comparative studies with other surgical procedures.

The duration of recommended follow-up will depend on the likely timing of anticipated or potential adverse outcomes

I think it's really important that IFSO regulates this. There are a lot of surgeons inventing new procedures with no or little regulations.

The procedure to be compared against depends on the nature of the new procedure. 
In the absence of any established criteria, the process has thus far been somewhat arbitrary with potential for conflict of opinion amongst the societies themselves. For example, though One Anastomosis (Mini) Gastric Bypass
(OAGB/MGB) is listed as an accepted procedure on the IFSO website [7] and there is a position statement approved by British Obesity and Metabolic Surgery Society in the United Kingdom [8], many other national societies including

Table 7 A Brief Questionnaire with Summary of Responses

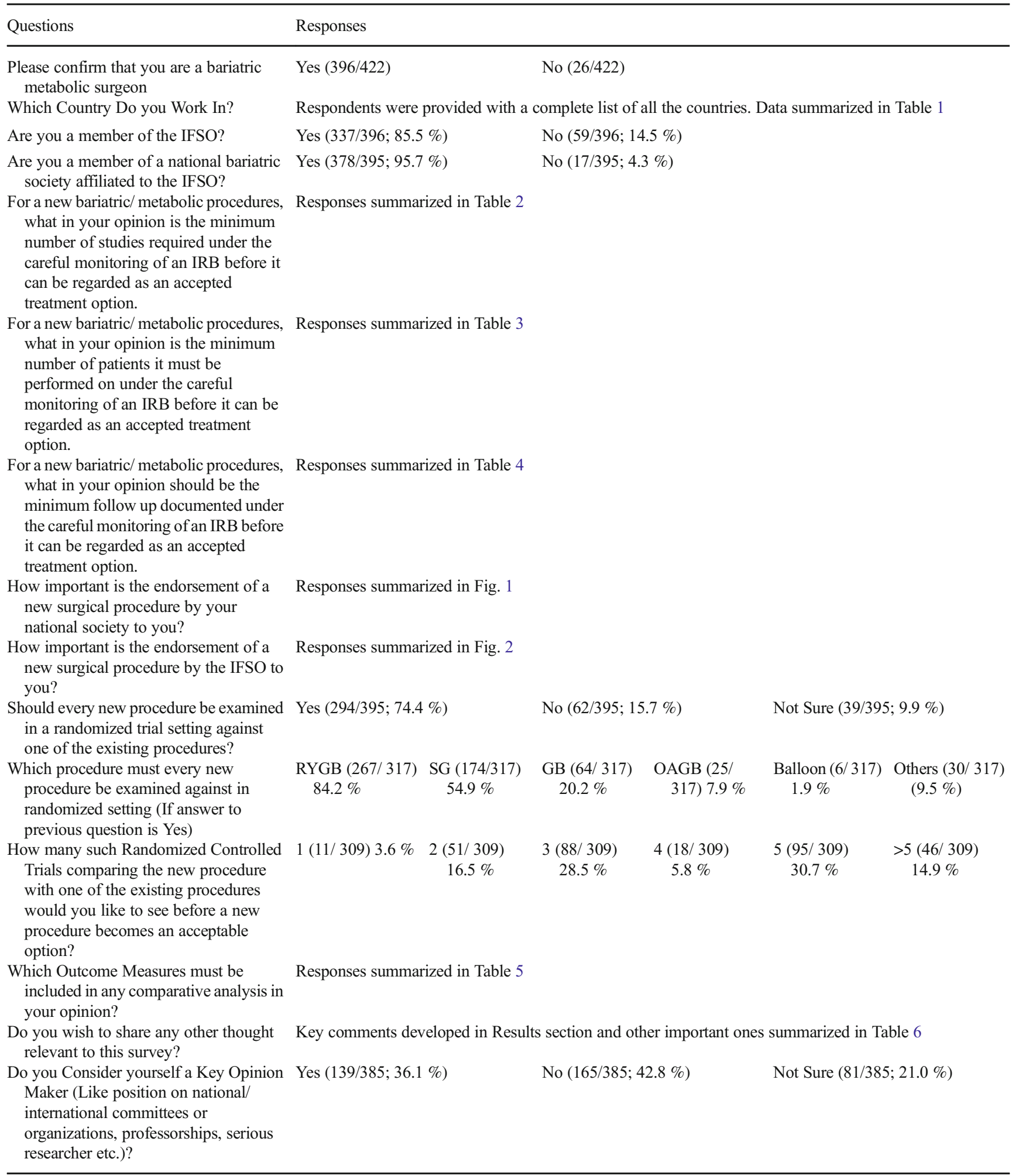


the American Society for Metabolic and Bariatric Surgery [9] do not currently include it in their list of bariatric surgical procedures.

The requirement of 5 clinical studies on 500 patients with 5 years follow-up, as well as the need for randomized comparisons, would appear reasonable and adequate to most bariatric and metabolic surgeons. Though majority of the surgeons indicated that they would like to see at least 4-5 such randomized comparisons, one has to bear in mind this probably indicates the ground reality when most current randomized clinical trials involving the discipline have small sample sizes $[10,11]$. In this context, even one large multicentre trial should be acceptable. Several respondents highlighted this point. IFSO and national societies could play a role in organizing such trials.

Though, the findings of this survey could only realistically apply to future procedures, they might also allow for better benchmarking in areas of current clinical controversy [12] and difference of opinions. Similarly, though this survey focuses on bariatric surgical procedures, it cannot be impossible to come up with indicative numbers for endoscopic procedures.

This survey focuses on defining criteria for new bariatric procedures rather than technical modification or improvisation of existing ones [13]. Though the definition of what should constitute a new procedure itself can be arbitrary [14, 15] and has been one of the biggest stumbling blocks in developing a clear framework for surgical innovation, generally if an operation is different enough from the existing alternatives to need a new name, it could safely be categorized as a new procedure. At the same time, one recognizes that major alterations in even existing procedures itself should probably go through a process of closer monitoring. At the very least, there should be an expectation that surgeons document their variation, audit their results and share the findings with others. Similarly, when surgeons are developing a new approach (i.e., robotic or natural orifice) for an existing operation, it is likely that a different set of rules will be required - those that primarily govern the safety of that approach in general and specifically for the procedure under consideration.

It has been suggested [16] that it is incumbent upon bariatric surgeons to 'compare and contrast' the new technology and procedures against the 'standards of care'. In this survey too, most respondents felt every new procedure should be examined against one of the existing alternatives in a randomized setting. Most surgeons felt RYGB $(n=267,84.2 \%)$ and SG $(n=174,54.9 \%)$ were most suitable for such comparisons.

It is also worth pointing out only two surgeons in this survey felt that appropriate animal models should be developed first before human trials are conducted. Indeed, it is not easy to carry out animal research in accordance with the ARRIVE guidelines [17] and the findings may well not be extrapolatable to human populations. At the same time, development of appropriate animal models of bariatric procedures is necessary to understand the underlying mechanisms and physiology [18, 19] and facilitate the development of less invasive treatment options in the future. This does not, however, have to happen before the procedure is introduced into routine clinical practice but may be advisable.

There are several weaknesses to this survey. It is a survey of bariatric surgeons on bariatric surgical procedures. The findings cannot hence be extrapolated to other surgical procedures and other (endoscopic) bariatric procedures. This survey is specific to bariatric surgeons.

Since the link to the survey was widely popularized, we are unable to define a response rate for our survey. However, authors cannot think of any possible bias as a result of it especially because of its relatively large sample size. The survey was conducted in English language and hence excludes non-English speaking surgeons. This problem is however not confined to this survey and can also be traced to other areas of scientific communications.

Patient safety and innovation are not mutually exclusive. The pace, as well as the acceptability of any surgical innovation, can be enhanced if there are appropriate mechanisms in place. Clearly-defined processes and procedures will enable innovators and protect patients. This survey should be seen as an attempt to understand the popular opinion of bariatric surgeons on what constitutes an experimental or new procedure and at what stage can a procedure be recommended for inclusion into mainstream clinical practice. Individual procedures will have unique characteristics that will demand modification of these criteria. The findings of this survey should hence only be used for the purposes of guiding authoritative committees of national and international societies, and for future consensus building exercises. It is worth emphasizing the difference between a survey and a consensus statement here [20]. This survey of bariatric surgeons does not attempt to build a consensus. Any consensus building exercise will necessarily have to start with identifying a multidisciplinary group of experts with an interest in this area followed by adoption of an agreed modified Delphi protocol [20]. There is no reason why that cannot be the next step for the bariatric community in this difficult area.

It is possible that the opinions expressed in this survey were influenced by the choices offered. The choices were however constructed by the authors as the most logical options for each question. Moreover, respondents were allowed to disagree with the choices offered through the provision of a free text box to enter 'other' choices. Authors would also like to caution against overinterpretation of survey findings. The findings of this survey should simply form a starting point of discussion in this somewhat controversial and difficult area of debate. They are not meant to influence ground reality until an international consensus can be reached amongst experts. 


\section{Conclusion}

Most bariatric surgeons feel that any new bariatric procedure must first be evaluated in at least 5 clinical studies, conducted under the strict monitoring of Institutional Review Boards, on 500 or more patients with minimum documented follow-up of 5 years. Majority also felt the need for randomized comparisons with one of the existing alternatives like Roux-en-Y Gastric Bypass or Sleeve Gastrectomy. At the same time, we must be cautious in interpreting the findings of a grassroots survey like this. The findings of this survey are simply aimed at starting a discussion on this topic and cannot be used to influence the ground reality until an international consensus can be reached amongst experts.

IFSO, International Federation for the Surgery of Obesity and Metabolic Disorders; ASMBS, American Society for Metabolic and Bariatric Surgery; IRB, Institutional Review Board; OAGB/MGB, One Anastomosis (Mini) Gastric Bypass; RYGB, Roux-en-Y Gastric Bypass; SG, Sleeve Gastrectomy; GB, Gastric Banding.

Author Contribution KM conceived the idea for the topic, conducted the survey, and wrote most of the manuscript. All authors participated in the discussions on the topic. All authors have seen the final version and approve of it.

\section{Compliance with Ethical Standards}

Conflicts of Interest None of the authors have any conflict of interest to declare.

Source(S) of Funding None.

Statement of Human and Animal Rights Not Applicable.

Statement of Informed Consent Not Applicable.

\section{References}

1. Johnson J, Rogers W. Innovative surgery: the ethical challenges. J Med Ethics. 2012;38(1):9-12.

2. Hopkins J, Howes N, Chalmers K, et al. By-band trial management group. What are important outcomes of bariatric surgery? An in- depth analysis to inform the development of a core outcome set and a comparison between the views of surgeons and other health professionals (the BARIACT study). Lancet. 2015;385(Suppl 1):S43.

3. Dixon JB, Logue J, Komesaroff PA. Promises and ethical pitfalls of surgical innovation: the case of bariatric surgery. Obes Surg. 2013;23(10):1698-702.

4. Shikora SA. A call for maintaining ethical behavior in bariatric surgery. Obes Surg. 2012;22(6):849-50.

5. Hofmann B. Stuck in the middle: the many moral challenges with bariatric surgery. Am J Bioeth. 2010;10(12):3-11.

6. Caniano DA. Ethical issues in pediatric bariatric surgery. Semin Pediatr Surg. 2009;18(3):186-92.

7. No Authors Listed. One Anastomosis Gastric Bypass. http://www. ifso.com/one-anastomosis-gastric-bypass. Accessed 8 March 2016.

8. No Authors Listed. BOMSS Position Statement on Mini Gastric Bypass. http://www.bomss.org.uk/wp-content/uploads/2014/09 /BOMSS-MGB-position-statement-September-20141.pdf. Accessed 8 March 2016.

9. No Authors Listed. Bariatric Surgery Procedures. American Society for Metabolic and Bariatric Surgery. http://asmbs. org/patients/bariatric-surgery-procedures. Accessed 8 March 2016.

10. Lee WJ, Yu PJ, Wang W, et al. Laparoscopic roux-en-Y versus mini-gastric bypass for the treatment of morbid obesity: a prospective randomized controlled clinical trial. Ann Surg. 2005;242(1): 20-8.

11. Sharma S, Narwaria M, Cottam DR, et al. Randomized doubleblinded trial of laparoscopic gastric imbrication $\mathrm{v}$ laparoscopic sleeve gastrectomy at a single Indian institution. Obes Surg. 2015;25(5):800-4.

12. Mahawar KK, Jennings N, Brown J, et al. "mini" gastric bypass: systematic review of a controversial procedure. Obes Surg. 2013;23(11):1890-8.

13. Guarner V. Improvising, innovating and experimenting in surgery. A serious problem without ethical regulation in current surgical practices. Gac Med Mex. 2008;144(5):445-8.

14. Reitsma AM, Moreno JD. Ethical regulations for innovative surgery: the last frontier? J Am Coll Surg. 2002;194(6):792-801.

15. Reitsma AM, Moreno JD. Ethics of innovative surgery: US surgeons' definitions, knowledge, and attitudes. J Am Coll Surg. 2005;200(1):103-10.

16. Raper SE, Sarwer DB. Informed consent issues in the conduct of bariatric surgery. Surg Obes Relat Dis. 2008;4(1):60-8.

17. NC3Rs Reporting Guidelines Working Group. Animal research: reporting in vivo experiments: the ARRIVE guidelines. J Physiol. 2010;588(Pt 14):2519-21.

18. Del Castillo Déjardin D, Sabench Pereferrer F, et al. The evolution of experimental surgery in the field of morbid obesity. Obes Surg. 2004;14(9):1263-72.

19. Rao RS, Rao V, Kini S. Animal models in bariatric surgery-a review of the surgical techniques and postsurgical physiology. Obes Surg. 2010;20(9):1293-305.

20. Mahawar KK, Aggarwal S, Carr WR, et al. Consensus statements and bariatric surgery. Obes Surg. 2015;25(6):1063-5. 